\title{
Erratum to: Magnetic ionic liquid-assisted synthesis of polyaniline/AgCl nanocomposites by interface polymerization
}

\author{
Qiang Zhang $\cdot$ Fangjun Liu $\cdot$ Liang Li • \\ Guoliang Pan $\cdot$ Songmin Shang
}

Published online: 9 December 2010

(C) Springer Science+Business Media B.V. 2010

\section{Erratum to: J Nanopart Res DOI 10.1007/s11051-010-0070-8}

Author would like to change Fig. 1b as given below:

In "Results and discussion" section sentence starting with "When the content of PVP..." should read "When the content of PVP was increased to 4\%, the particles with a shell thickness of 10-20 nm and a core diameter of 30-60 $\mathrm{nm}$ were prepared".

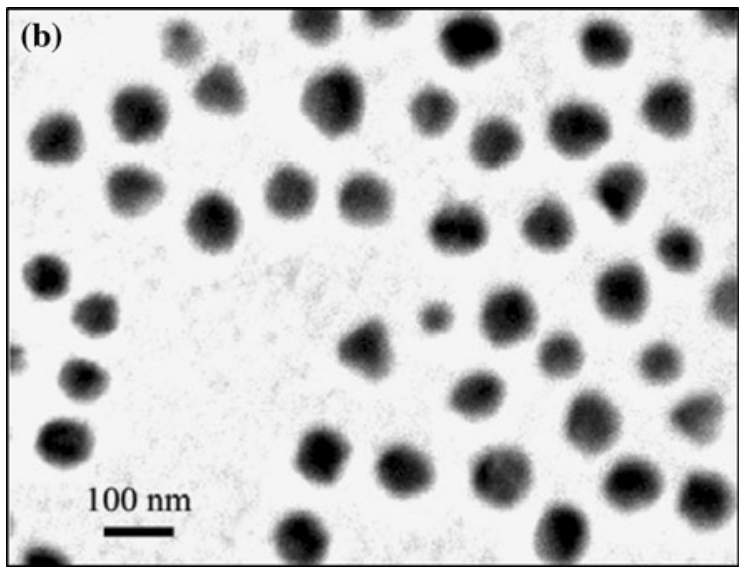

Fig. 1 TEM images of $\mathrm{PANi} / \mathrm{AgCl}$ nanocomposites synthesized at different concentrations of PVP: a $0.5 \%$ and b $4 \%$
The online version of the original article can be found under doi:10.1007/s11051-010-0070-8.

\section{Q. Zhang · F. Liu · L. Li ( $₫) \cdot$ G. Pan}

Key Laboratory for Green Chemical Process of Ministry of Education, School of Materials Science and Engineering, Wuhan Institute of Technology, Wuhan 430073, People's Republic of China e-mail: msell08@163.com

S. Shang Institute of Textiles and Clothing, The Hong Kong Polytechnic University, Hung Hom, Kowloon, Hong Kong, People's Republic of China 